\title{
Learning Analytics aus institutioneller Perspektive: Ein Orientierungsrahmen für die hochschulische Datennutzung
}

\section{Falk Scheidig und Monika Holmeier}

\section{Zusammenfassung}

Der digitale Wandel führt zu einer Zunahme des Datenvolumens und neuen Möglichkeiten der Datennutzung. Auch an Hochschulen befördert der voranschreitende Technologieeinsatz in Studium und Lehre die als „Datafication“ beschriebene Entwicklung. Hieraus erwachsen einerseits Anforderungen bezüglich des kompetenten Umgangs mit Daten (Data Literacy) und insbesondere des Datenschutzes, denen sich Hochschulen schon heute nicht entziehen können, andererseits aber auch neue Potenziale der gezielten Datennutzung. Unter „Learning Analytics“ werden seit etwa einer Dekade Möglichkeiten der Datenauswertung entwickelt und diskutiert, die ein tieferes Verständnis und eine Optimierung von Lernumgebungen und -prozessen in Aussicht stellen und sowohl Dozierende und Studierende als auch hochschulische Leitungspersonen und -gremien adressieren. Ungeachtet der technischen Dimension der Datenverarbeitung ist die Implementierung von Learning Analytics vor allem auch organisational anspruchsvoll. Zugleich korrespondieren mit Szenarien hochschulischer Datennutzung begründete Einwände und ethische Bedenken. Im Beitrag wird zunächst in das noch junge Feld Learning Analytics eingeführt und anschließend ein Orientierungsrahmen für Hochschulen vorgelegt,

F. Scheidig $(\bowtie) \cdot$ M. Holmeier

Pädagogische Hochschule FHNW, Windisch, Schweiz

E-Mail: falk.scheidig@ @hnw.ch

M. Holmeier

E-Mail: monika.holmeier@fhnw.ch 
der aus institutioneller Perspektive 25 Handlungsanforderungen für einen sensiblen, potenzialorientierten und organisationsbewussten Umgang mit lehr- und lernbezogenen Daten beschreibt.

\section{Schlüsselwörter}

Learning Analytics • Educational Data Literacy • Hochschulentwicklung • Datennutzung $\bullet$ Datenschutz $\bullet$ Governance in Higher Education Institutions $\bullet$ Data-Driven Decision Making

\section{Datafication of Higher Education}

Der mit dem digitalen Wandel voranschreitende Technologieeinsatz führt in vielen Bereichen zur massiven Zunahme von Daten - so auch im Bildungswesen (Jarke und Breiter 2019). Insbesondere Hochschulen stellen aufgrund des gewachsenen Stellenwerts digitaler Lehr- und Lernszenarien (Technology-Enhanced Learning, TEL) und der Etablierung hochschulweiter Tools wie Campus-ManagementSystemen zur Studierenden- und Lehrangebotsverwaltung sowie Lernplattformen (Learning-Management-Systemen, LMS) datenreiche Institutionen dar. Die als „Datafication of Higher Education“ (Selwyn und Gašević 2020) gefasste Entwicklung besitzt multiple Implikationen: Hochschulen müssen verantwortungsvoll mit „ihren“ Daten umgehen und unter anderem gestiegenen Anforderungen des Datenschutzes entsprechen. Zugleich eröffnen sich neue Möglichkeiten, die Daten zur Informationsgewinnung und für datengestützte Entscheidungen (Data-Driven Decision Making) zu nutzen.

In diesem Kontext entfaltete sich seit circa 2010 ein internationaler Diskurs zu Learning Analytics, der sich primär auf den Hochschulbereich zentriert. Buckingham Shum und McKay (2018, S. 26) umreißen den Kern dieser Bestrebungen wie folgt: „The focus of learning analytics is the application of analytics approaches to gain insight into educational data to improve teaching and learning". Im viel rezipierten Horizon Report, der jährlich aktuelle und prognostizierte Trends technologiegestützten Lehrens und Lernens vorstellt, wird Learning Analytics regelmäßig als bedeutsamer Entwicklungsbereich aufgeführt (zum Beispiel jüngst Brown et al. 2020). Im Gegensatz etwa zu den USA, Kanada, Australien oder China erhielt dieses noch junge Feld in Europa - von Ausnahmen abgesehen, zum Beispiel in den Niederlanden - bislang eher wenig Aufmerksamkeit (Nouri et al. 2019).

Mit Learning Analytics verbinden sich einerseits große Erwartungen, etwa eine Reduktion von Studienabbrüchen, die Personalisierung des Lernens oder ein 
effizienterer Ressourceneinsatz, andererseits aber auch fundamentale pädagogische, ethische und datenschutzrechtliche Vorbehalte. Slade und Prinsloo (2013) verweisen beispielsweise aus einer soziokritischen Perspektive auf die durch Datenanalysen evozierten Machtfragen und Überwachungstendenzen. Sie argumentieren aber zugleich, dass sich Hochschulen den aus Daten erwachsenden Potenzialen kaum entziehen können: „Ignoring information that might actively help to pursue an institution's goals seems shortsighted to the extreme. Institutions are accountable, whether it is to shareholders, to governments, or to students themselves“ (Slade und Prinsloo 2013, S. 1521). Es existieren verschiedene Aktivitäten und Initiativen, die auf eine rechtskonforme, werteorientierte und transparente Datennutzung zielen und zum Beispiel unter ,Trusted Learning Analytics“ (Drachsler und Greller 2016; Hansen et al. 2020) firmieren. Hieran anknüpfend gibt der Beitrag nachfolgend eine konzise Einführung ${ }^{1} \mathrm{zu}^{\mathrm{Le}}$ earning Analytics im Hochschulbereich unter besonderer Gewichtung der institutionellen Perspektive. Den beiden Prämissen folgend, dass mit Learning Analytics aufgeworfene Herausforderungen vielfach auch für bereits seit Längerem an Hochschulen praktizierte Formen der Datenerfassung und -verwendung gelten und dass sich jenseits der konkreten Datenverarbeitung vor allem gewichtige organisationale Fragen stellen, mündet der Beitrag in die Formulierung eines Orientierungsrahmens mit 25 Handlungsanforderungen für den Umgang mit hochschulischen Daten (Abschn. 4), der den hier skizzierten Potenzialen und Herausforderungen (Abschn. 2 und 3) Rechnung trägt.

\section{Grundlagen, Potenziale und Limitationen von Learning Analytics}

Die Datennutzung im Kontext von Lehre und Studium ist Hochschulen prinzipiell nicht fremd, so werden etwa seit geraumer Zeit Daten für institutionelle Steuerungsprozesse verwendet oder auch studentische Leistungen auf der Ebene einzelner Lehrveranstaltungen von Dozierenden, wenngleich oftmals unstrukturiert, für ein tieferes Verständnis von Lernprozessen und zur Weiterentwicklung von Lehrveranstaltungen ausgewertet. Mit Learning Analytics assoziierte Innovationspotenziale beziehen sich auf die umfassende und systematische Erschließung vorhandener und neuer Datenquellen sowie neue, mitunter komplexe Formen der Datenauswertung, wie sie aufgrund limitierter Datenverarbeitungskapazitäten vor

\footnotetext{
${ }^{1}$ Einen umfassenderen Zugang bieten unter anderem die Systematic Reviews von Leitner, Khalil und Ebner (2017) sowie Misiejuk und Wasson (2017).
} 
wenigen Jahren nicht oder nur mit sehr großem Aufwand möglich waren (Prinsloo 2019). Learning-Analytics-Aktivitäten bewegen sich damit an der Schnittstelle von Informatik, angewandter Statistik, empirischer Bildungs- und Hochschulforschung, Hochschuldidaktik und TEL, besitzen aber auch Bezüge zu Business Analytics/Business Intelligence, Bildungsmonitoring und Qualitätsmanagement (Scheidig 2019).

Zentrale Learning-Analytics-Ressource sind in den meisten Fällen Daten über Studierende (zum Beispiel demografische Angaben) oder von Studierenden (zum Beispiel Lernaktivitäts- und Leistungsdaten). Es lassen sich prinzipiell drei Arten der Datengewinnung unterscheiden (Open Knowledge Foundation 2013): erstens das Lokalisieren relevanter Daten in intern vorliegenden Datensets (zum Beispiel Noten oder ECTS-Punkte aus dem Campus-Management-System), zweitens das Lokalisieren und Anfordern von externen Daten (zum Beispiel Daten zur Bibliotheksnutzung aus einem Verbundsystem oder Daten von übergeordneten Behörden) und drittens das Erheben von Daten (zum Beispiel mittels Befragung oder elektronischer Erfassung). Die meisten bei Learning-Analytics-Aktivitäten genutzten Daten liegen bereits hochschulintern vor, entsprechen also erstgenannter Kategorie. Hier handelt es sich häufig um automatisiert erfasste Daten, zum Beispiel Aktivitätsdaten von LMS (zum Beispiel Loginzeit, Nutzungsdauer, Interaktionsintensität, Pfade, Aufgabenperformance, Diskussionsaktivitäten, Ratings) oder auch bei Massive Open Online Courses (MOOCs), E-Assessments und Webseitenaufrufen gespeicherte Daten. Daneben finden sich zahlreiche Beispiele für die Analyse weiterer Daten, so etwa GPS- und Bluetooth-Daten von studentischen Mobiltelefonen (Kassarnig et al. 2017), Daten der Campuskarte für die Bibliotheks-, Mensa- oder Kopiergerätnutzung (Ram et al. 2015) oder Daten aus Video- und Mikrofonaufzeichnungen (Ochoa und Worsley 2016). Mit Lai und Schildkamp (2013, S. 11) lässt sich eine kategoriale Differenzierung vornehmen zwischen Input-Daten (zum Beispiel Studierenden- und Dozierendenmerkmale), Outcome-Daten (zum Beispiel Prüfungs- und Evaluationsergebnisse), Prozessdaten (zum Beispiel zu Instruktionen und Lernpfaden) und Kontextdaten (zum Beispiel zu Curricula und Infrastrukturnutzung). Des Weiteren kann zwischen statischen, (eher) zeitüberdauernden Daten (zum Beispiel Herkunft, bildungsbiografischer Status) und dynamischen Daten (zum Beispiel Lernaktivität, Motivation, Emotion) unterschieden werden, ferner zwischen strukturierten Daten (zum Beispiel im Vorlesungsverzeichnis erfasste Module) und unstrukturierten Daten (etwa Wortmeldungen im LMS-Forum). 
Wie bei der Datengewinnung lassen sich auch verschiedene Arten der Datenauswertung unterscheiden. Das Spektrum erstreckt sich von der Datenvisualisierung und ,konventionellen“ statistischen Verfahren über Soziale-NetzwerkAnalysen bis hin zu Machine Learning und Künstlichen Neuronalen Netzwerken. Auswertungen vorliegender Publikationen zeigen, dass vor allem deskriptive Statistik (zum Beispiel Häufigkeit, Mittelwert, Streuung) und inferenzstatistische Verfahren (zum Beispiel Regression) dominieren und demgegenüber MachineLearning- oder Text-Mining-Verfahren eher selten angewendet werden (Leitner et al. 2017; Misiejuk und Wasson 2017). Die Wahl des analytischen Vorgehens korrespondiert mit den verfügbaren Daten sowie mit den Zielen und Fragestellungen der Datenanalyse, die sich hinsichtlich des Anspruchsniveaus differenzieren lassen; Norris und Baer (2013) verorten in ihrem achtstufigen Learning-AnalyticsImplementierungsmodell beispielsweise standardisierte Berichte wie Kennzahlenund Monitoringberichte auf der untersten Stufe, statistisch tendenziell anspruchsvollere Prognosen (Predictive Modeling) hingegen auf der zweithöchsten und präskriptive Datenauswertungen (Optimization) auf der höchsten Stufe.

Die mittels Learning Analytics gewonnenen Informationen können unterschiedliche Hochschulakteur*innen adressieren, typische Beispiele sind Dashboards (Datencockpits) für Leitungspersonen, Kursmonitoringsysteme für Dozierende (zum Beispiel LMS-Plugins mit Analytics-Elementen) oder Informationssysteme für Studierende (Scheidig 2019). Diese Tools können verschiedene Funktionen erfüllen, zum Beispiel Daten visualisieren, Vergleiche vornehmen, Feedback geben, Empfehlungen formulieren oder Prognosen zu Lern- und Studienerfolg berechnen (Clow 2013; Greller und Drachsler 2012; Ifenthaler und Widanapathirana 2014). Ifenthaler (2015) hat ausgehend von einer Unterscheidung summativer (oder retrospektiver), Echtzeit- sowie prognostischer Analysen eine Learning-Analytics-Nutzenmatrix vorgelegt, in der für verschiedene Anspruchsgruppen die möglichen Erträge der drei Analyseformen mit Beispielen benannt werden (Tab. 1).

Die mit Learning Analytics eingenommene Perspektive besitzt gleichwohl Limitationen, die mitunter strukturell bedingt und im Fachdiskurs wenig strittig sind. Die Zentrierung auf Studierendendaten und studentische Aktivitätsdaten wird problematisch, wenn beispielsweise bei kleinen Studierendenkohorten kaum Referenzdaten bestehen oder wenn digitale Lehr- und Lernformen, zum Beispiel der LMS-Einsatz, schwach ausgeprägt sind. Damit geht einher, dass die Fokussierung auf datengenerierende Facetten von Studium und Lehre einseitige Wahrnehmungen befördern kann, wenn nur das in den Blick gerät, was mittels Daten ausgeleuchtet wird (,Streetlight Effect“). Des Weiteren stellen die meisten Daten nur Momentaufnahmen dar, von denen nicht prinzipiell auf überdauernde 


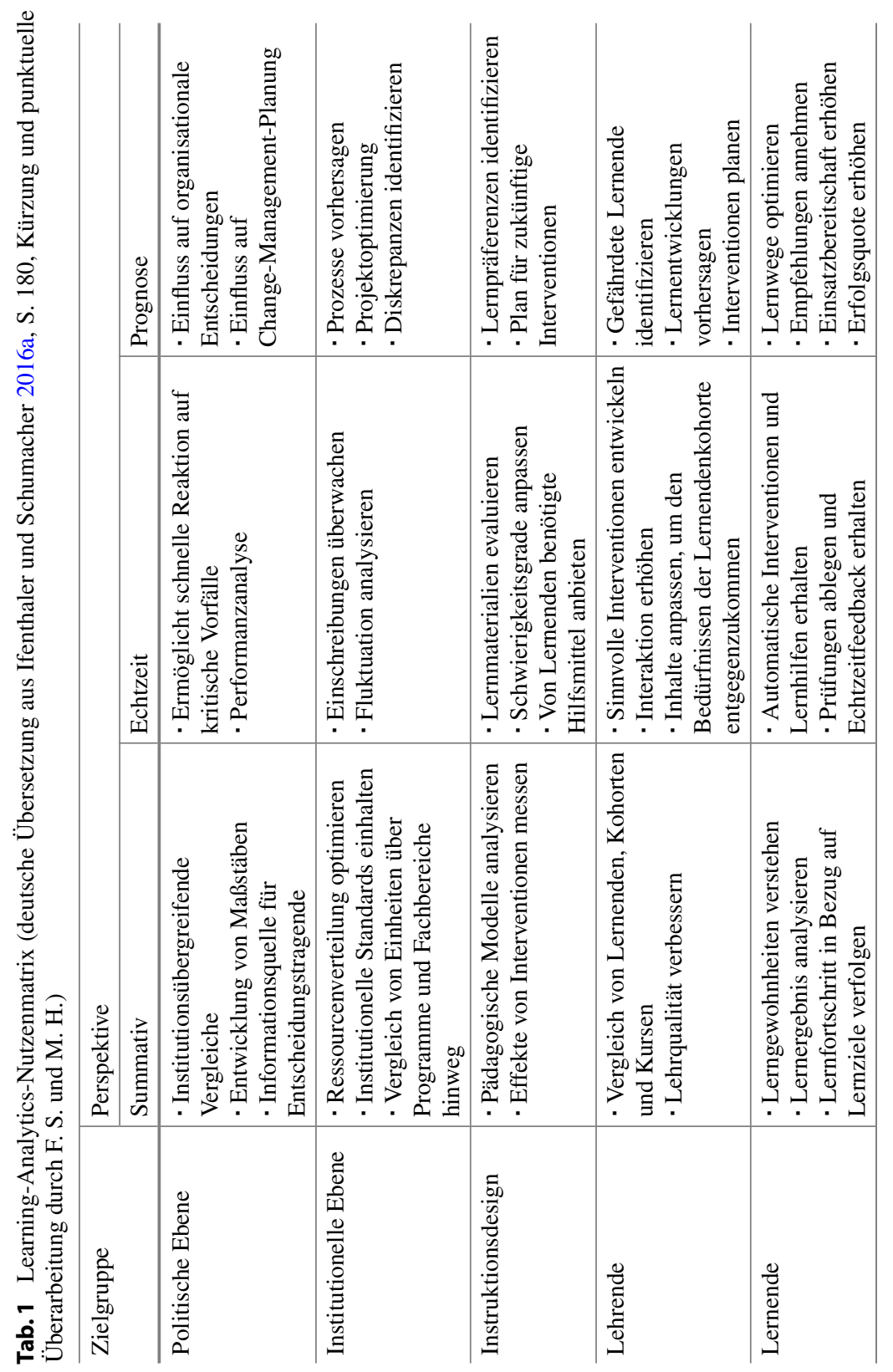


Muster im Sinne einer Fortschreibung der Bildungsbiografie geschlossen werden kann (Slade und Prinsloo 2013). Die Analyse studentischen Verhaltens in LMS wird ferner als behavioristischer Zugang eingeordnet und in seiner quantitativen Ausrichtung problematisiert, weil damit keine näheren qualitativen Einsichten in Lernprozesse geliefert würden (Long und Siemens 2011). Die meisten Analysen führen demgemäß auch nicht zu Erklärungs- oder gar Handlungswissen, womit der ambitionierte Anspruch der Optimierung ${ }^{2}$ des Lernens und der Lernumgebungen vielfach auf die Bereitstellung von potenziell handlungsrelevanten Informationen schrumpft (Scheidig, im Erscheinen). Kritik bezieht sich schließlich auch auf die bislang unzureichende Verknüpfung von Learning Analytics mit pädagogischer Theorie (Marzouk et al. 2016).

\section{$3 \quad$ Herausforderungen der Datennutzung}

Die Implementierung von Learning Analytics stellt auf verschiedenen Ebenen hohe Anforderungen an den Umgang mit Daten (Data Literacy) - von der Datenbeschaffung und -aufbereitung über die Datenauswertung und Bereitstellung der Ergebnisse bis hin zur Dateninterpretation und datengestützten Ableitung von Maßnahmen (Ridsdale et al. 2015; Schüller et al. 2019). Viele Hochschulen verfügen im Hinblick auf die konkrete Datenverarbeitung nicht über hinreichende personelle und infrastrukturelle Ressourcen (etwa Data Scientists, Datenbanken), wie Ifenthaler (2017) in einer internationalen Studie konstatiert.

Nicht minder anspruchsvoll sind die Anforderungen an den Datenschutz und ethisches Handeln. In einschlägigen Learning-Analytics-Frameworks (für eine Übersicht vgl. Leitner et al. 2019) wird datenschutzbezogenen und ethischen Geboten sowie potenziellen Risiken besondere Aufmerksamkeit geschenkt (zum Beispiel Ferguson et al. 2016; Drachsler und Greller 2016; Pardo und Siemens 2014; Slade und Prinsloo 2013). Im Hinblick auf den Datenschutz betreffen zentrale Aspekte die Zweckbindung der Datennutzung und das Prinzip der Datensparsamkeit, die Klärung und Transparenz in Bezug auf Datenspeicherung, -löschung, -zugriff und -eigentum, die Gewährleistung informationeller Selbstbestimmung und das aktive Einholen des Einverständnisses (Informed Consent), die

\footnotetext{
${ }^{2}$ Den Optimierungsanspruch dokumentiert beispielsweise das prominenteste Definitionsangebot von Learning Analytics: „Learning analytics is the measurement, collection, analysis and reporting of data about learners and their contexts, for purposes of understanding and optimising learning and the environments in which it occurs" (Long und Siemens 2011, S. 34).
} 
Deidentifizierung von Personen durch Anonymisierung, Pseudonymisierung oder Aggregierung, die Datensicherheit und das Vorbeugen von Datenmissbrauch.

Von diesen - unter anderem in der seit Mai 2018 geltenden europäischen Datenschutzgrundverordnung (DSGVO) aufgegriffenen - Anforderungen abzugrenzen sind ethische Aspekte der Datennutzung. Diese betreffen etwa Bedenken hinsichtlich einer Kontrollatmosphäre (,gläserne Studierende“), der Beförderung sozialen Drucks (zum Beispiel durch Vergleiche), möglichen Normierungstendenzen, der Neutralität von Daten(auswertungen) sowie der Intransparenz von entscheidungsfundierenden Algorithmen. Zum Teil bestehen ethische Dilemmata, etwa das Spannungsverhältnis zwischen der Möglichkeit, frühzeitig potenziell abbruchgefährdete Studierende zu identifizieren, um auf kritisches Studierverhalten aufmerksam zu machen oder die Betroffenen individuell zu unterstützen, und der Gefahr, hierdurch prinzipiell zukunftsoffene Lernprozesse zu präjudizieren oder im Sinne einer selbst erfüllenden Prophezeiung durch demotivierende Prognoseinformationen einen Studienabbruch herbeizuführen. West et al. (2016) beschreiben einen Prozess, wie derlei komplexe ethische Fragen im Kontext von Learning Analytics einer Bearbeitung zugeführt werden können; die vier darin beschriebenen Etappen fassen sie wie folgt zusammen: „(1) Explore the issue, (2) apply an institutional lens to the issue, (3) view the alternative actions in light of the ethical theoretical approaches, (4) document the decision made". Für den deutschen Hochschulraum haben Hansen et al. (2020) einen Verhaltenskodex für „Trusted Learning Analytics“ vorgelegt, der sieben leitende Prinzipien für Learning Analytics umfasst: (1) Verbesserung der Bedingungen für Lernen und Lehren, (2), Unterstützungsangebot für alle Studierende, (3) transparenter Umgang mit Daten, (4) kritischer Umgang mit Daten, (5) menschliche Kontrolle, (6) Führungsverantwortung, (7) Verpflichtung zu Weiterbildungsangeboten.

Als eine entscheidende Gelingensbedingung - insbesondere im Umgang mit datenschutzbezogenen und ethischen Herausforderungen - wird die Partizipation zentraler Stakeholder ausgewiesen, dies gilt vor allem für den Einbezug der Studierenden, die das Gros der verarbeiteten Daten generieren und direkt oder indirekt durch das Leitmotiv der Weiterentwicklung von Studium und Lehre adressiert werden (Ifenthaler und Schumacher 2016b). In einer Studie zu studentischen Erwartungen an Learning Analytics arbeiteten Schumacher und Ifenthaler (2018) heraus, dass Studierende Datenanalysen vor allem dann akzeptieren, wenn sie hierdurch Impulse zur Selbstüberprüfung mit Blick auf den Lernstand und die Erreichung von Lernzielen sowie Empfehlungen für das weitere Lernen im Rahmen des Lehrangebots erhalten. Das Involvieren von Stakeholdern verweist dabei wieder auf den institutionellen Kontext und beschreibt eine Dimension der organisationalen Einbettung, wie sie im Folgenden thematisiert wird. 


\section{$4 \quad$ Orientierungsrahmen für die hochschulische Datennutzung}

Die vorliegenden Frameworks für Learning Analytics (zum Beispiel Drachsler und Greller 2016; Ferguson et al. 2016; Greller und Drachsler 2012; Pardo und Siemens 2014; Tsai und Gašević 2017) formulieren zentrale Herausforderungen zur Datennutzung und prononcieren insbesondere bedeutsame ethische und datenschutzrechtliche Fragen. Als generische und partiell nicht explizit auf den Hochschulkontext bezogene Orientierungsangebote liefern sie verschiedenen Akteur*innen im Bildungsbereich einen Überblick über die mit Learning Analytics verbundenen Anforderungen. Dies bedingt, dass die Unterscheidung zwischen der makro- und mikrodidaktischen Ebene nicht ausreichend zur Geltung gelangen kann. Ebenso befördert eine Gesamtbetrachtung von Learning Analytics, dass die notwendige Unterscheidung zwischen institutioneller Rahmensetzung (zum Beispiel Ausgestaltung einer Data Policy, Ziele und Grenzen der Datennutzung) und der konkreten Datenverarbeitung (zum Beispiel statistische Auswertung, Sicherstellen der Datensicherheit) wenig sichtbar wird. Damit geht einher, dass in den vorliegenden Frameworks die organisationale Perspektive keine explizite Hervorhebung erfährt, worin ein generelles Desiderat im Diskurs über Learning Analytics gesehen werden kann (Roberts et al. 2017). Dies wird hier zum Anlass genommen, aufbauend auf vorliegende Frameworks einen Orientierungsrahmen (Tab. 2) zu formulieren, der die institutionelle Perspektive von Hochschulen stärker gewichtet und primär - aber nicht ausschließlich - Leitungspersonen und -gremien sowie Verantwortliche im Wissenschaftsmanagement adressiert. Damit verbindet sich nicht der Anspruch, ein weiteres Framework vorzulegen, sondern datenbezogene Handlungsbereiche für Entscheidungstragende an Hochschulen zu kartieren und zu illustrieren, dass Learning Analytics über konkrete Datenverarbeitungsprozesse und Datenschutzrichtlinien hinausreicht.

Für die Erstellung des nachfolgenden Orientierungsrahmens wurde eine Synthese aus verschiedenen Frameworks (Drachsler und Greller 2016; Ferguson et al. 2016; Greller und Drachsler 2012; Pardo und Siemens 2014; Tsai und Gašević 2017) entlang gesamtinstitutioneller Fragestellungen vorgenommen und mit Elementen hochschulbezogener Implementierungs- und Projektmanagementstrategien verbunden. Wesenszug des Orientierungsrahmens ist nicht ein spezifischer Innovationsgehalt, sondern die akzentuierte institutionelle Optik in der Zuwendung zu Learning Analytics. Im Unterschied zu den genannten Frameworks und anderen Orientierungsangeboten zu Learning Analytics wird im Orientierungsrahmen eine gestufte Unterscheidung vorgenommen zwischen generellen Anforderungen in Bezug auf den Schutz und die Nutzung hochschulischer Daten, 
Tab. 2 Orientierungsrahmen für einen sensiblen, potenzialorientierten und organisationsbewussten Umgang mit lehr-/lernbezogenen Hochschuldaten. (Eigene Darstellung)

Datenschutz und institutionelles Datenbewusstsein

Data Policy im Einklang mit (inter)nationalen (darunter DSGVO, BDSG-neu) und weiteren (zum Beispiel föderalen) Datenschutzrichtlinien erarbeiten, fortlaufend aktualisieren, den

1 Anspruchsgruppen gegenüber klar kommunizieren, (mit Beispielen) erläutern und zugänglich machen; Datenmissbrauchsszenarien (etwa Diskriminierung) antizipieren und Vorkehrungen treffen

Inventur verfügbarer Daten und assoziierter Prozesse vornehmen (Data Map) und definieren, welche Daten wann, wo und wie erfasst, gespeichert, verarbeitet und weitergeleitet werden, wem sie gehören (Data Ownership), wer verantwortlich ist, wer Zugriffsrechte besitzt und ob die Daten anonymisiert, pseudonymisiert oder aggregiert werden; Rahmen für interne Datenrepositorien setzen (zum Beispiel für Forschungszwecke oder spätere (Re-)Analysen)

Rahmenbedingungen für dezentrale Aktivitäten der Datenerhebung und -verarbeitung

3 festlegen (inklusive Tabuzonen), zum Beispiel Entscheidung hinsichtlich des Zugriffs auf LMSDaten für Dozierende oder die Bereitstellung von Studienverlaufsdaten für die Studiengangsleitung/-koordination

Einverständnis (Opting-in), wo es erforderlich ist, auf Basis umfassender Information zur Datenverarbeitung und -speicherung einholen (Informed Consent); Widerspruchsmöglichkeit und deren Konsequenzen definieren und erklären; für einzelne Systeme und Prozesse im Rahmen der Data Policy Datenschutzerklärungen gestalten

Umsetzung der Data Policy sicherstellen; Systeme und Prozesse so konfigurieren, dass Datenschutzprinzipien eingehalten (Data Protection by Design) und nur notwendige Daten erfasst und gespeichert werden (Data Protection by Default); Einhaltung von Datenschutz und -sicherheit bei IT-Lösungen als relevantes Kriterium definieren (zum Beispiel hinsichtlich des Standorts externer Server oder einer Verpflichtung auf nationale Datenschutzrichtlinien)

6

Verantwortliche Stellen mandatieren, einrichten oder ihren Zuständigkeitsbereich ausweiten, zum Beispiel Datenschutzbeauftragte und Kontrollinstanzen auf verschiedenen Ebenen, Ombudsstelle bei Datenmissbrauchsverdacht

7 Meinungsbildung und das Treffen begründeter Entscheidungen unter anderem in Bezug auf die Implementierung von Learning Analytics Vertrauen gewinnen; Sensibilität beachten; datenbezogene Bedürfnisse, Haltungen, Bedenken klären und einbeziehen; Partizipation ermöglichen; geteilte Verantwortung im Umgang mit Potenzialen und Risiken der Arbeit mit Daten entwickeln

Datenschutz und -nutzung als dynamisches Feld verstehen und als Thema wiederkehrend aufgreifen, zum Beispiel bei Veränderung der rechtlichen oder institutionellen Rahmenbedingungen oder IT-Anpassungen, bei der Überarbeitung von internen Prozessen sowie als Prüfkriterium bei der Anschaffung neuer Tools

\section{Learning-Analytics-Aktivitäten}

10

Ziele und Zweck für Learning-Analytics-Aktivitäten bestimmen und Prioritäten setzen; Erwartungsmanagement vornehmen (zum Beispiel personalisiertes Lernen ermöglichen versus Ressourceneffizienz kontrollieren); Nutzungsgruppen definieren und involvieren (zum Beispiel Hochschulmanagement und -gremien, Dozierende, Studierende)

Fokus und Fragestellungen für Datenanalysen spezifizieren; Gegenstände für konkretes Datenmodell definieren (zum Beispiel Studienabbruch); konkrete, an den Zielen und Fragestellungen orientierte sowie überprüfbare Anforderungen an Learning-AnalyticsAktivitäten formulieren 
Tab. 2 (Fortsetzung)

2

Einbettung in den organisationalen Kontext vornehmen (Strategiekonformität), zum strategierelevanten Rahmen (etwa Leitbild, Lehrstrategie, Studienkonzept, E-Strategie) und zur Hochschulkultur und -steuerung (zum Beispiel Führungsphilosophie) ins Verhältnis setzen

Ressourceneinsatz für Learning Analytics definieren, insbesondere finanziellen Rahmen für Personal, IT-Anschaffungen und Programmierung (Initial- und laufende Kosten); kalkulierten Aufwand und erwarteten Ertrag gegenüberstellen; indirekte Kosten bedenken, etwa Zeiteinsatz durch die Erweiterung von Prozessen

Hochschulinterne Expertise im Hinblick auf Learning Analytics identifizieren, einbeziehen und vernetzen; institutionellen Kompetenzaufbau und organisationales Wissensmanagement fördern; gegebenenfalls weitere externe Ressourcen konsultieren, Good-Practice-Beispiele recherchieren, Netzwerk aufbauen

Möglichkeiten und Bedarf der Form und Frequenz der Datenauswertung und - bereitstellung klären, etwa automatisierte Echtzeitanalysen über ein eigenes Dashboard (zum Beispiel Studierendenkennzahlen), Integration in eine bestehende Systemlandschaft (etwa Software für Geschäftsprozesse), als Erweiterung eines Tools (zum Beispiel LMSPlugin) oder in Berichtsform (etwa Lehrbericht, Monitoringbericht); relevante Stichdaten definieren

Für Learning Analytics relevante Daten lokalisieren und systematisch sammeln; falls erforderlich neue Datenquellen erschließen und Daten von Dritten anfordern; Daten hinsichtlich ihrer Relevanz gemäß Zwecksetzung gewichten; Datenerfassung in Abhängigkeit von der Zielsetzung und den bereits bestehenden Daten anpassen (zum Beispiel differenzierter erfassen) oder limitieren

Voraussetzungen für einen zweckgebundenen und mit der Data Policy konformen Datenaustausch zwischen Organisationseinheiten schaffen (Data Fusion), zum Beispiel zwischen IT, Qualitätsmanagement, Controlling, dezentralen Stellen

Prozesse so einrichten, dass Datenqualität kontinuierlich geprüft und optimiert wird (zum Beispiel konsistente Datenstruktur); Datenkorrekturen ermöglichen; Loops vorsehen, sodass Anliegen der Datennutzenden an die Datengewinnung/-erfassung und -verarbeitung berücksichtigt werden können; bei datenbasierten Informationen die Datengrundlage und - verarbeitung explizieren (Nachvollziehbarkeit und Verständlichkeit sicherstellen, Einschätzung und Einordnung der Daten(auswertung) ermöglichen)

Strategische und operative Verantwortung für Learning-Analytics-Aktivitäten definieren (Project/Product/Process Owner); unter Umständen organisationale Anpassungen vornehmen (zum Beispiel Einrichten einer Stabseinheit oder eines Single Point of Contact für Learning Analytics)

Umgang mit den Ergebnissen und Folgemaßnahmen unter Beachtung der übergeordneten Zielsetzung der Learning-Analytics-Aktivitäten antizipieren, zum Beispiel Präsentation kritischer Werte gegenüber Studierenden, personalisierte Lernangebote, Beratungsangebote von abbruchgefährdeten Studierenden

Risiken (zum Beispiel falsch-positive oder falsch-negative Analysen), nicht intendierte Wirkungen (zum Beispiel selbst erfüllende Prophezeiung demotivierender

21 Studienerfolgsprognosen), ethische Implikationen (etwa Atmosphäre der Überwachung), Limitationen (zum Beispiel begrenzte Aussagekraft von LMS-Daten) und künftige (Um-) Nutzung (zum Beispiel für Indikatorensteuerung) reflektieren

Learning-Analytics-Prozesse modellieren; Prozessgovernance, -dokumentation und - lebenszyklus definieren; Schritt vom Projektstatus zu einer nachhaltigen institutionellen vollziehen und Learning Analytics in bestehende Prozesse integrieren, zum Beispiel Führungsprozesse, Qualitätsmanagement, Lehrangebotsplanung, Budgetprozess, Studiengangsentwicklung („Meaningful Data“) 
Tab. 2 (Fortsetzung)

23

Institutionelle Data Literacy stärken, sodass elementare statistische Begriffe und Konzepte angemessen verstanden, Daten adäquat interpretiert, zielorientiert genutzt und zugleich kritisch hinterfragt werden; Bereitschaft für datenbasiertes Handeln fördern

24

Evaluation von Learning-Analytics-Aktivitäten durchführen; Erreichen der formulierten Ziele und Einhalten der Data Policy prüfen; den Grad datengestützten Handelns reflektieren

25

Vertrauen und Akzeptanz durch transparente Kommunikation der Learning-AnalyticsAktivitäten gewinnen (zum Beispiel auf Websites, in einem Jahresbericht)

die unabhängig von Learning-Analytics-Aktivitäten bestehen, und jenen Anforderungen, die mit Learning-Analytics-Vorhaben hinzutreten oder an Bedeutung gewinnen. Diese Abgrenzung ist keineswegs trennscharf - beispielsweise ist die Steigerung der Datenqualität (Punkt 18) grundsätzlich erstrebenswert -, soll aber verdeutlichen, dass die meisten in einschlägigen Frameworks genannten Fragestellungen und Herausforderungen nicht genuin auf Learning Analytics bezogen sind, sondern auch für die bereits an Hochschulen praktizierte Datenerfassung, -speicherung und -verarbeitung gelten und insofern obligatorischer Art sind. Im Zentrum des Orientierungsrahmens steht das Bemühen um einen sensiblen, potenzialorientierten und organisationsbewussten Umgang mit lehr-/ lernbezogenen Hochschuldaten: sensibel insofern, als datenschutzrechtliche und ethische Belange beachtet und auch die Ansprüche der Mitarbeitenden bedacht werden sollten; potenzialorientiert, weil zu fragen ist, welche Ressourcen, welches Wissen und Können und vor allem auch welche Daten an der Institution vorhanden sind, und nicht allein danach, welche Risiken minimiert werden müssen; organisationsbewusst in der Hinsicht, dass auch auf eine gemeinsame Haltung der Institution und auf ein gemeinsames Verständnis hingearbeitet werden sollte.

Die einzelnen Punkte des Orientierungsrahmens besitzen zahlreiche Querbezüge untereinander, beanspruchen keine Vollständigkeit und bedürfen einer Übersetzung und Konkretisierung vor dem Hintergrund der Spezifika und Anforderungen des jeweiligen Hochschulkontexts und des jeweils fokussierten Learning-Analytics-Schwerpunkts. Aufgrund der Betonung der institutionellen Perspektive finden Aspekte der angewandten Datenverarbeitung - zum Beispiel die sehr zeitaufwendigen Schritte der Datenaufbereitung (Romero et al. 2014) - im Orientierungsrahmen keine Beachtung, obschon ihre Bedeutung nicht in Abrede gestellt wird. Die Punkte sind nicht gewichtet, ihre Reihenfolge suggeriert eine durchaus intendierte Prozesshaftigkeit, wenngleich die Punkte nicht streng chronologisch zu interpretieren sind, da erstens viele Schritte nicht zuletzt aufgrund ihrer Interdependenz in der Hochschulpraxis parallel verlaufen 
und miteinander verwoben sind und zweitens die meisten Schritte kontinuierliche Aufgaben beschreiben oder zumindest einer regelmäßigen Vergewisserung bedürfen.

\section{$5 \quad$ Fazit}

Die Auseinandersetzung mit hochschulischen Daten erlangte in den vergangenen Jahren gesteigerte Aufmerksamkeit und könnte künftig weiter an Bedeutung gewinnen, weil das Datenvolumen zunehmen wird und sich mit den Daten schon heute verschiedene und mitunter konkurrierende Interessen verbinden. Hochschulen können sich diesem Thema nicht verwehren - etwa Datenschutzgebote ignorieren - und sind gefordert, im Umgang mit „ihren“ Daten kompetent, rechtskonform und sensibel zu agieren. Aus der Zunahme des Datenvolumens erwachsen aber nicht nur Anliegen an Datenschutz und -sicherheit, sondern auch neue Potenziale der Datenanalyse für informationsgestütztes Hochschulmanagement und hochschuldidaktische Zwecke. Hochschulen sollten für sich, aber auch gegenüber ihren Anspruchsgruppen klären, wie sie mit den vorliegenden Daten und damit korrespondierenden Potenzialen und Risiken umgehen. So wäre unter anderem denkbar, dass angesichts von Studienabbruchquoten, die in Deutschland und im OECD-Durchschnitt bei ca. $30 \%$ rangieren (Neugebauer et al. 2019, S. 1031 f.), der Druck auf Hochschulen zunimmt, die ihnen bereits zur Verfügung stehenden Daten aus Campus- und Lern-Management-Systemen zu nutzen, um Studierende bedarfsgerechter zu unterstützen und abbruchgefährdete Studierende frühzeitig zu identifizieren. Zugleich verdienen insbesondere die organisationalen und ethischen Implikationen solcher oder ähnlicher Datennutzungsszenarien eine eingehende Berücksichtigung. Die hier formulierten 25 Handlungsanforderungen sollen diesbezüglich eine erste Orientierung stiften und unterstreichen die vielfältigen institutionellen Herausforderungen im Horizont der „Datafication“. Der begründeten Entscheidung, ob und wie Hochschulen Learning-AnalyticsVorhaben verfolgen - oder unter welchen Bedingungen dies auf nachgeordneten Organisationsebenen gestattet wird -, sollte in jedem Fall eine multiperspektivische Auseinandersetzung mit dem Für und Wider sowie den Limitationen von Datenanalysen und den damit assoziierten institutionellen Anforderungen vorausgehen. 


\section{Literatur}

Brown, M., McCormack, M., Reeves, J. D. Brooks, C., \& Grajek, S. (2020). EDUCAUSE Horizon Report. Teaching and Learning Edition. Louisville, CO: Educause. https://lib rary.educause.edu/-/media/files/library/2020/3/2020_horizon_report_pdf.pdf. Zugegriffen: 20. Mai. 2020.

Buckingham Shum, S., \& McKay, T. (2018). Architecting for learning analytics: Innovating for sustainable impact. Educause Review, 53(2), 25-37.

Clow, D. (2013). An overview of learning analytics. Teaching in Higher Education, 18(6), 683-695. https://doi.org/10.1080/13562517.2013.827653.

Drachsler, H., \& Greller, W. (2016). Privacy and analytics: It's a DELICATE issue: A checklist to establish trusted learning analytics. In Proceedings of the 6th international conference on learning analytics and knowledge (S. 89-96). New York: ACM. https://doi.org/10. $1145 / 2883851.2883893$.

Ferguson, R., Hoel, T., Scheffel, M., \& Drachsler, H. (2016). Guest editorial: Ethics and privacy in learning analytics. Journal of Learning Analytics, 3(1), 5-15. https://doi.org/ 10.18608/jla.2016.31.2.

Greller, W., \& Drachsler, H. (2012). Translating learning into numbers: A generic framework for learning analytics. Educational Technology \& Society, 15(3), 42-57.

Hansen, J., Rensing, C., Herrmann, O., \& Drachsler, H. (2020): Verhaltenskodex für Trusted Learning Analytics. Entwurf für die hessischen Hochschulen. Frankfurt a. M.: Innovationsforum Trusted Learning Analytics 2020. https://www.dipfdocs.de/volltexte/2020/ 18903/pdf/Hansen_Rensing_Herrmann_Drachsler_2020_Verhaltenskodex_Trusted_L earning_Analytics_A.pdf. Zugegriffen: 20. Mai 2020.

Ifenthaler, D. (2015). Learning analytics. In J. M. Specter (Hrsg.), The SAGE encyclopedia of educational technology, 2 (S. 447-451). Thousand Oaks: Sage.

Ifenthaler, D. (2017). Are higher education institutions prepared for learning analytics? TechTrends, 61(4), 366-371. https://doi.org/10.1007/s11528-016-0154-0.

Ifenthaler, D., \& Schumacher, C. (2016a). Learning Analytics im Hochschulkontext. WiSt - Wirtschaftswissenschaftliches Studium, 4, 172-177.

Ifenthaler, D., \& Schumacher, C. (2016b). Student perceptions of privacy principles for learning analytics. Educational Technology Research and Development, 64(5), 923-938. https://doi.org/10.1007/s11423-016-9477-y.

Ifenthaler, D., \& Widanapathirana, C. (2014). Development and validation of a learning analytics framework: Two case studies using support vector machines. Technology, Knowledge and Learning, 19(1-2), 221-240. https://doi.org/10.1007/s10758-014-9226-4.

Jarke, J., \& Breiter, A. (2019). Editorial: The datafication of education. Learning, Media and Technology, 44(1), 1-6. https://doi.org/10.1080/17439884.2019.1573833.

Kassarnig, V., Bjerre-Nielsen, A., Mones, E., Lehmann, S., \& Lassen, D. D. (2017). Class attendance, peer similarity, and academic performance in a large field study. PloS one, 12(11), (e0187078). https://doi.org/10.1371/journal.pone.0187078.

Lai, M. K., \& Schildkamp, K. (2013). Data-based decision making: An overview. In K. Schildkamp, M. K. Lai, \& L. Earl (Hrsg.), Data-based decision making in education: Challenges and opportunities (S. 9-12). Dordrecht: Springer. 
Leitner, P., Ebner, M., \& Ebner, M. (2019). Learning analytics challenges to overcome in higher education institutions. In D. Ifenthaler, D.-K. Mah \& J. Y.-K. Yau (Hrsg.), Utilizing learning analytics to support study success (S. 91-104). Cham: Springer. https://doi.org/ 10.1007/978-3-319-64792-0_6.

Leitner, P., Khalil, M., \& Ebner, M. (2017). Learning analytics in higher education - A literature review. In A. Peña-Ayala (Hrsg.), Learning analytics: Fundaments, applications, and trends: A view of the current state of the art to enhance E-learning (S. 1-23). Cham: Springer. https://doi.org/10.1007/978-3-319-52977-6_1.

Long, P., \& Siemens, G., (2011). Penetrating the fog: Analytics in learning and education. Educause Review, 46(5), 31-40. https://er.educause.edu/articles/2011/9/penetrating-thefog-analytics-in-learning-and-education. Zugegriffen: 20. Mai 2020.

Marzouk, Z., Rakovic, M., Liaqat, A., Vytasek, J., Samadi, D., Stewart-Alonso, J., Ram, I., Woloshen, S., Winne, P. H., \& Nesbit, J. C. (2016). What if learning analytics were based on learning science? Australasian Journal of Educational Technology, 32(6), 1-18. https:// doi.org/10.14742/ajet.3058.

Misiejuk, K., \& Wasson, B. (2017). State of the Field Report on Learning Analytics. SLATE Report 2017-2. Bergen: Centre for the Science of Learning \& Technology (SLATE). https://bora.uib.no/bitstream/handle/1956/17740/SoF\%20Learning\%20A nalytics\%20Report.pdf?sequence=1\&isAllowed=y. Zugegriffen: 20. Mai 2020.

Neugebauer, M., Heublein, U., \& Daniel, A. (2019). Studienabbruch in Deutschland: Ausmaß, Ursachen, Folgen, Präventionsmöglichkeiten. Zeitschrift für Erziehungswissenschaft, 22, 1025-1046. https://doi.org/10.1007/s11618-019-00904-1.

Norris, D. M., \& Baer, L. L. (2013). Building Organizational Capacity for Analytics. https:// library.educause.edu/-/media/files/library/2013/2/pub9012-pdf.pdf. Zugegriffen: 20. Mai 2020.

Nouri, J., Ebner, M., Ifenthaler, D., Saqr, M., Malmberg, J., Khalil, M., Bruun, J., Viberg, O., Conde González, M. Á., Papamitsiou, Z., \& Berthelsen, U. D. (2019). Efforts in Europe for data-driven improvement of education - A review of learning analytics research in seven countries. International Journal of Learning Analytics and Artificial Intelligence for Education, 1 (1), 8-27. https://doi.org/10.3991/ijai.v1i1.11053.

Ochoa, X., \& Worsley, M. (2016). augmenting learning analytics with multimodal sensory data. Journal of Learning Analytics, 3(2), 213-219. https://doi.org/10.18608/jla.2016. 32.10 .

Open Knowledge Foundation. (2013). Data Wrangling Handbook. Release 0.1. https://readth edocs.org/projects/datapatterns/downloads/pdf/latest/. Zugegriffen: 20. Mai 2020.

Pardo, A., \& Siemens, G. (2014). Ethical and privacy principles for learning analytics. British Journal of Educational Technology, 45(3), 438-450. https://doi.org/10.1111/bjet.12152.

Prinsloo, P. (2019). A social cartography of analytics in education as performative politics. British Journal of Educational Technology, 50(6), 2810-2823. https://doi.org/10.1111/ bjet.12872.

Ram, S., Wang, Y., Currim, F., \& Currim, S. (2015). Using big data for predicting freshmen retention. In 2015 international conference on information systems: Exploring the information frontier, ICIS 2015. Association for information systems. https://aisel.aisnet.org/ icis2015/proceedings/DecisionAnalytics/13/. Zugegriffen: 20. Mai 2020. 
Ridsdale, C., Rothwell, J., Smit, M., Bliemel, M., Irvine, D., Kelley, D., Matwin, S., Wuetherick, B., \& Ali-Hassan, H. (2015). Strategies and best practices for data literacy education knowledge synthesis report. https://doi.org/10.13140/RG.2.1.1922.5044.

Roberts, L. D., Chang, V., \& Gibson, D. (2017). Ethical considerations in adopting a universityand system-wide approach to data and learning analytics. In B. Kei Daniel (Hrsg.), Big data and learning analytics in higher education: Current theory and practice (S. 89-108). Cham: Springer. https://doi.org/10.1007/978-3-319-06520-5_7.

Romero, C., Romero, J. R., \& Ventura, S. (2014). A survey on pre-processing educational data. In A. Peña-Ayala (Hrsg.), Educational Data Mining. Studies in Computational Intelligence, 524 (S. 29-64). https://doi.org/10.1007/978-3-319-02738-8_2.

Scheidig, F. (2019). Learning Analytics: Gegenstand, Potentiale, Herausforderungen. In B. Berendt, A. Fleischmann, N. Schaper, B. Szczyrba, M. Wiemer \& J. Wildt (Hrsg.), Neues Handbuch Hochschullehre. Ergänzungsband Nr. 93 (S. 65-96). Berlin: DUZ.

Scheidig, F. (im Erscheinen). Von datengestützter Optimierung und gläsernen Studierenden: Beispiele, Potentiale und Probleme von Learning Analytics. In Deutsche Gesellschaft für Hochschuldidaktik (Hrsg.), Regeneration Hochschullehre. Bielefeld: wbv media.

Schüller, K., Busch, P., \& Hindinger, C. (2019). Future Skills: Ein Framework für Data Literacy - Kompetenzrahmen und Forschungsbericht (Arbeitspapier, 47). Berlin: Hochschulforum Digitalisierung. https://doi.org/10.5281/zenodo.3349865.

Schumacher, C., \& Ifenthaler, D. (2018). Features students really expect from learning analytics. Computers in Human Behavior, 78, 397-407. https://doi.org/10.1016/j.chb.2017. 06.030 .

Selwyn, N., \& Gašević, D. (2020). The datafication of higher education: Discussing the promises and problems. Teaching in Higher Education, 25(4), 527-540. https://doi.org/ 10.1080/13562517.2019.1689388.

Slade, S., \& Prinsloo, P. (2013). Learning analytics: ethical issues and dilemmas. American Behavioral Scientist, 57(10), 1510-1529. https://doi.org/10.1177/0002764213479366.

Tsai, Y. S., \& Gašević, D. (2017). Learning analytics in higher education - challenges and policies: A review of eight learning analytics policies. In Proceedings of the Seventh International Learning Analytics \& Knowledge Conference (S. 233-242). New York: ACM. https://doi.org/10.1145/3027385.3027400.

West, D., Huijser, H., \& Heath, D. (2016). Putting an ethical lens on learning analytics. Educational Technology Research and Development, 64(5), 903-922. https://doi.org/10. 1007/s11423-016-9464-3. 
Open Access Dieses Kapitel wird unter der Creative Commons Namensnennung 4.0 International Lizenz (http://creativecommons.org/licenses/by/4.0/deed.de) veröffentlicht, welche die Nutzung, Vervielfältigung, Bearbeitung, Verbreitung und Wiedergabe in jeglichem Medium und Format erlaubt, sofern Sie den/die ursprünglichen Autor(en) und die Quelle ordnungsgemäß nennen, einen Link zur Creative Commons Lizenz beifügen und angeben, ob Änderungen vorgenommen wurden.

Die in diesem Kapitel enthaltenen Bilder und sonstiges Drittmaterial unterliegen ebenfalls der genannten Creative Commons Lizenz, sofern sich aus der Abbildungslegende nichts anderes ergibt. Sofern das betreffende Material nicht unter der genannten Creative Commons Lizenz steht und die betreffende Handlung nicht nach gesetzlichen Vorschriften erlaubt ist, ist für die oben aufgeführten Weiterverwendungen des Materials die Einwilligung des jeweiligen Rechteinhabers einzuholen. 